“C2020 IEEE. Personal use of this material is permitted. Permission from IEEE must be obtained for all other uses, in any current or future media, including reprinting/republishing this material for advertising or promotional purposes, creating new collective works, for resale or redistribution to servers or lists, or reuse of any copyrighted component of this work in other works." 


\title{
Low-Cost All-Metal Resonant-Cavity Antenna for High Power Applications
}

\author{
Foez Ahmed*, Student Member, IEEE, Muhammad U. Afzal* ${ }^{*}$ Member, IEEE, Touseef Hayatt*, Student Member, IEEE, \\ and Karu P. Esselle*†, Fellow Member, IEEE \\ ${ }^{*}$ School of Engineering, Macquarie University, Sydney, NSW-2109 \\ ${ }^{\dagger}$ School of Electrical and Data Engineering, University of Technology Sydney, Sydney, NSW-2007 \\ foez.ahmed@students.mq.edu.au
}

\begin{abstract}
Low-cost all-metal partially reflecting surface (AM PRS) based resonant-cavity antennas are presented in this paper. The AM PRSs are made by introducing square-shaped slots in a thin metallic sheet having moderate to high reflectivity ranging from $-4.12 \mathrm{~dB}$ to $-1.27 \mathrm{~dB}$. An RCA designed using highly reflecting AM PRS has maximum directivity of $16.54 \mathrm{dBi}$ and low $3 \mathrm{~dB}$ directivity bandwidth of $\mathbf{9 . 1 7 \%}$ whereas those designed using less reflective AM PRSs have high directivity bandwidth but smaller peak directivity within the operating band.
\end{abstract}

Index Terms-All-metal partially reflecting surface; resonantcavity antenna; low-cost antennas.

\section{INTRODUCTION}

Low-cost planar high-gain front-end antennas are essential for a long-distance communication such as point-to-point microwaves links, and satellite reception etc. The resonant-cavity antennas (RCAs) have been a great interest of the research community due to their amazing features like low-profile, single feed system and highly directive far-field radiation [1]. In 1956, the concept of Resonant-Cavity antennas was proposed using of partially reflecting superstrate (PRS) placed above the feed antennas [2]. Since then, RCAs have been extensively investigated to improve various design aspects such as enhancing gain bandwidth and increasing maximum far-field gain. The PRSs used in RCAs can be 1D [3], 2D or $3 \mathrm{D}$ periodic structures and conventionally engineered using commercially available copper cladding dielectric laminates or thick dielectrics [4].

Despite being actively researched, one of the limitations with RCAs is that they are not cost-effective, which is mainly due to the use of commercial costly laminates. The excessive use of dielectric also prohibitively limits their use for highpower microwave applications. To address these challenges, this work presents preliminary results on the development of all-metal PRSs (AM PRSs) that are made using thin sheets of metal. Three different RCAs are designed, using AM PRSs having varying levels of reflection, and their crucial far-field radiation characteristics including peak directivity and the $3 \mathrm{~dB}$ directivity bandwidth are discussed in the paper.

The rest of the paper is organized as follows. Section II explains the design methodology of RCAs having AM PRSs. A comparative study among three different RCAs including

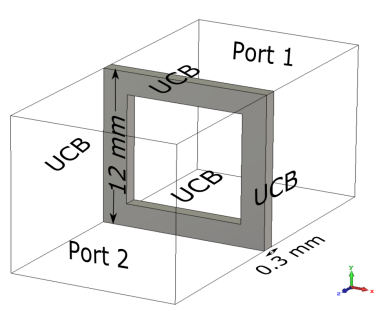

(a)

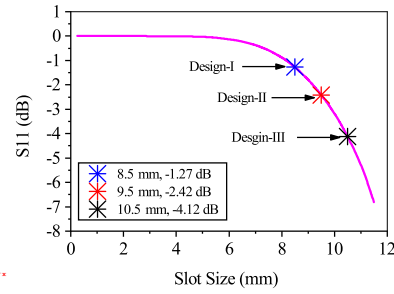

(b)
Fig. 1. (a) Dimensional details of Unit Cell and (b) $|S 11|$ at $11 \mathrm{GHz}$.

their far-field radiation characteristics is presented in section III. Finally, concluding remarks are in section IV.

\section{All-Metal PRS AND RCA}

A conventional RCA comprises a feed antenna backed by reflecting ground plane and a partially reflecting surface (PRS) or superstrated. The PRS is suspended above the ground plane so that a cavity, typically air-filled, is formed between the PRS and the ground plane. The feed excites the cavity at the centre of its aperture that resonates at the operating frequency and enhances far-field directivity by increasing the radiating aperture. The PRS is made of a large number of repeating periodic features, which are referred to as a unit cell. For the proposed PRS, the unit cell is made of all-metal sheet depicted in Fig. 1(a). It is made from a thin metal sheet having a squareshaped slot in the centre. The periodicity of the unit cell is $0.44 \lambda_{0}$ whereas the metal thickness is only $0.01 \lambda_{0}\left(\lambda_{0}\right.$ is the wavelength at the design frequency of $11 \mathrm{GHz}$ ).

The unit-cell is simulated with periodic boundary conditions in the lateral directions and the reflections are observed at the input port for the direction of wave propagation along the $\mathrm{z}$ axis. The size of the square-shaped slot is varied between 0.5 $\mathrm{mm}$ to $11.5 \mathrm{~mm}$ and the magnitude of the reflection coefficient is recorded, which is plotted in Fig. 1(b). It is visible that as the slot size increases, the reflection coefficient magnitude reduces significantly and vice versa. To understand the effect of AM PRS on the performance of an RCA, we selected three different unit cells having slot sizes $8.5 \mathrm{~mm}, 9.5 \mathrm{~mm}$ and $10.5 \mathrm{~mm}$ with reflection magnitude of $-1.27 \mathrm{~dB},-2.42 \mathrm{~dB}$, 


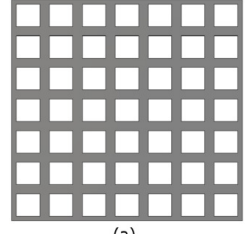

(a)

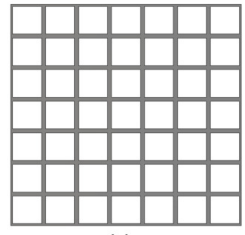

(c)

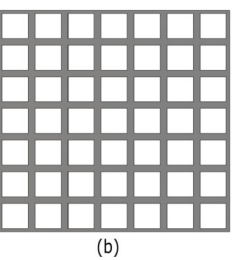

AM PRS

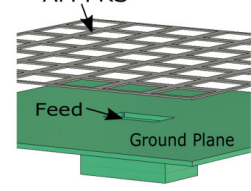

(d)
Fig. 2. Top view of AM PRS for (a) Design-I, (b) Design-II, (c) Design-III and (d) perspective view of an RCA.

and $-4.12 \mathrm{~dB}$, respectively. Three RCAs designed using PRSs having high, moderate, and low reflections are referred to as Design-I, Design-II, and Design-III, respectively which are pictorially shown in Fig. 2(a)-(c). A perspective view of one of the RCAs is shown in Fig. 2(d). The primary feed is a slotted waveguide that has a base (or ground plane) of $9.5 \lambda_{0} \mathrm{~mm}^{2}$.

\section{Antenna PERformance}

The three antennas were simulated with CST Microwave Studio with full-wave simulations. The far-field pattern characteristics inducing directivity bandwidth and radiation pattern were investigated. The degree of reflectivity of AM PRS has significant effects on all-metal RCAs' performance. To explain this, the far-field pattern cuts of the three designs (Design I-III) taken in two principle planes; E- and H-planes at the operating frequency of $11 \mathrm{GHz}$ are plotted in Fig. 3. The Design I, that uses AM PRS having most reflection coefficient has the largest broadside directivity of $16.54 \mathrm{dBi}$ and the least $3 \mathrm{~dB}$ beamwidth of $23.9^{\circ}$ at E-plane. The peak broadside directivity of Design III is $12.4 \mathrm{dBi}$, which uses AM PRS having the least reflection coefficient magnitude. The Design II has an intermediate directivity value with a $3 \mathrm{~dB}$ beamwidth of $28.4^{\circ}$ at E-plane. The pattern quality in both principle planes for all three designs is acceptable with the highest sidelobe levels of $-17.1 \mathrm{~dB}$ that is observed in H-plane for Design-I.

Another, important design consideration of RCAs is the $3 \mathrm{~dB}$ directivity bandwidth. To understand the behaviour of the AM RCAs, we considered their radiation performance in a frequency band around the centre operating frequency of $11 \mathrm{GHz}$. The variation in broadside directivity for the three designs is pictorially presented in Fig. 4 . The $3 \mathrm{~dB}$ directivity bandwidth of Design I is the smallest and is 9.2\% (from 10.4 $\mathrm{GHz}$ to $11.4 \mathrm{GHz}$ ) of the centre frequency. The $3 \mathrm{~dB}$ directivity bandwidth, in terms of the percentage of central frequency, for Design-II and Design III is $9.61 \%$ (from $10.4 \mathrm{GHz}$ to 11.45 $\mathrm{GHz}$ ) and $19.82 \%$ (from $10.0 \mathrm{GHz}$ to $12.2 \mathrm{GHz}$ ), respectively. The directivity bandwidth is inversely proportional to the reflection magnitude of the AM PRS used for the RCA while

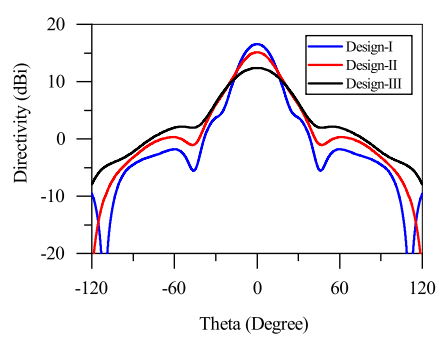

(a)

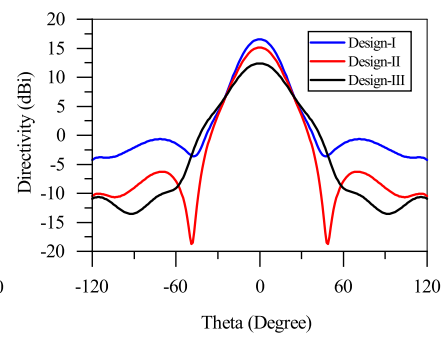

(b)
Fig. 3. Far-field pattern cuts of the three AM RCAs in (a) E- and (b) H-planes.

the highest directivity within the $3 \mathrm{~dB}$ bandwidth is directly proportional to the reflection magnitude of the AM PRS - a trade-off requires in between $3 \mathrm{~dB}$ directivity bandwidth and low peak gain and vice versa. Therefore, a higher reflective AM PRS can be designed for RCAs needed for narrowband and high-gain wireless applications whereas low reflective AM PRS based RCA can be used for medium-gain wideband applications.

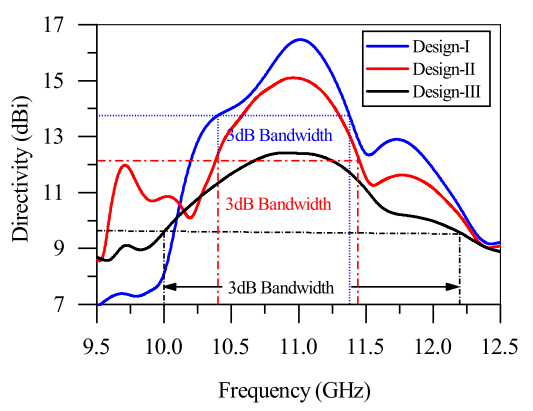

Fig. 4. Predicted directivity bandwidth of Design-I, Design-II and Design-III.

\section{CONCLUSION}

All-metal RCAs are studied and their significant results are presented. AM PRS based RCA with an aperture size of $9.5 \lambda_{0} \mathrm{~mm}^{2}$ can provide directivity between $12.4 \mathrm{dBi}$ to $16.54 \mathrm{dBi}$, using AM PRSs having a low-to-high magnitude of the reflection coefficient. The AM RCA can be developed at an extremely low-cost as they do not use any commercial dielectric laminates and are extremely useful for low-cost wireless applications.

\section{REFERENCES}

[1] A. P. Feresidis and J. C. Vardaxoglou, "High gain planar antenna using optimised partially reflective surfaces," IEE Proceedings: Microwaves, Antennas and Propagation, 2001.

[2] G. Von Trentini, "Partially Reflecting Sheet Arrays," IRE Transactions on Antennas and Propagation, 1956.

[3] A. R. Weily, K. P. Esselle, T. S. Bird, and B. C. Sanders, "Dual resonator 1-D EBG antenna with slot array feed for improved radiation bandwidth," IET Microwaves, Antennas and Propagation, 2007.

[4] A. A. Baba, R. M. Hashmi, K. P. Esselle, and A. R. Weily, "Compact high-gain antenna with simple all-dielectric partially reflecting surface," IEEE Transactions on Antennas and Propagation, vol. 66, no. 8, pp. 4343-4348, 2018 